\section{LONG-TERM OUTCOME OF ACUTE ENCEPHALITIS IN CHILDHOOD}

\author{
A. Fowler ${ }^{1}$, T. Stödberg ${ }^{2}$, M. Eriksson ${ }^{3}$, \\ R. Wickström² \\ ${ }^{1}$ Dept of Women's and Children's Health, \\ Karolinska Institute, ${ }^{2}$ Neuropediatric Unit, \\ ${ }^{3}$ Pediatric Infectious Disease Unit, Karolinska \\ University Hospital, Stockholm, Sweden
}

Background and aims: Short-term follow-up after childhood encephalitis shows persisting symptoms in $30-60 \%$. The aim of this study was to investigate the long-term outcome of childhood encephalitis and to find prognostic factors.

Methods: Out of 93 children treated for acute encephalitis between 2000-2004, 71 were eligible for follow-up. A structured interview, using two questionnaires, was conducted with the parents. Fifteen of the children with the most severe symptoms at the time of discharge also underwent an EEG-recording and tests of reaction time and working memory.

Results: Persisting symptoms were reported by $54 \%$. The predominant symptoms were personality changes and cognitive problems. Children who made a complete recovery did so within 6-12 months. The only significant risk factor for sequelae was severity of the disease leading to admission to the ICU. The risk for subsequent epilepsy was increased for children with seizures at presentation. Most follow-up EEGs showed improvement, but in $9 / 15$ it had not normalized. Children with encephalitis had slower reaction time compared to controls but no difference in working memory could be seen.

Conclusion: Persisting symptoms after childhood encephalitis are present in a substantial number of children. Seizures increased the risk for subsequent epilepsy; the only other prognostic marker was admission to the ICU. Even children who were considered fully recovered at discharge reported persisting symptoms at follow-up and children who made a full recovery did so within 6-12 months. We suggest that all children with encephalitis should be followed for one year after the acute illness.

\section{DEVELOPMENT OF CHILDREN WITH INTRACRANIAL HEMORRHAGE BORN AFTER VACUUM EXTRACTION}

\author{
M. Schot, F. Halbertsma, T. Katgert, L. Bok \\ Maxima Medisch Centrum, Veldhoven, \\ The Netherlands
}

Subject of the study: We describe the long term follow-up of a cohort of children with an intracranial hemorrhage $(\mathrm{ICH})$ associated with vacuum extraction.

Methods: We retrospectively searched our database from 1999 to 2007. Patient outcome was evaluated using the Bayley Scale of Infant Development (BSID-II-NL). Development was considered severely impaired if the scores were less than 55 , moderately impaired if they were 55 to 69 , and mildly impaired if they were 70 to 84 . If a child did not complete the BSID-II-NL, a pediatrician estimated the child's developmental level.

Results: We identified 25 patients with $\mathrm{ICH}$ after vacuum extraction. Eighteen patients were male. Mean gestational age was 39 weeks. Mean birth weight was 3283 grams. Clinical symptoms suspect for $\mathrm{ICH}$ varied among patients. Seizures were most frequently reported $(60 \%)$. Subarachnoid hemorrhage was most frequently seen $(n=12)$.

Motor development was severely impaired in four children(16\%), moderately impaired in one child (4\%) and mildly impaired in six children (24\%). Mental development was severely impaired in two children $(8 \%)$, moderately impaired in one child $(4 \%)$ and mildly impaired in seven children (28\%).

Conclusion: Delayed development is common in children with $\mathrm{ICH}$ after vacuum extraction. When there is a symptomatic $\mathrm{ICH}$ after vacuum extraction, long term follow-up is justified.

Predictive factors for neurodevelopmental delay such as Apgarscores, gestational age or birth weight could not be deducted from our series. 